\title{
High level of miR-221/222 confers increased cell invasion and poor prognosis in glioma
}

Chunzhi Zhang ${ }^{1,2,3 \dagger}$, Junxia Zhang ${ }^{2,3,4 \dagger}$, Jianwei Hao ${ }^{2,3 \dagger}$, Zhendong Shi ${ }^{2,3 \dagger}$, Yingyi Wang ${ }^{4}$, Lei Han ${ }^{2}$, Shizhu Yu ${ }^{3}$, Yongping You ${ }^{4}$, Tao Jiang ${ }^{5}$, Jinhuan Wang ${ }^{1}$, Meili Liu ${ }^{6}$, Peiyu Pu ${ }^{2,3}$ and Chunsheng Kang ${ }^{2,3,7^{*}}$

\begin{abstract}
Background: MiR-221 and miR-222 (miR-221/222), upregulated in gliomas, can regulate glioma cell cycle progression and apoptosis, respectively. However, the association of miR-221/222 with glioma cell invasion and survival remains unknown.

Methods: Invasion capability of miR-221/222 was detected by mutiple analyses, including diffusion tensor imaging (DTI), transwell, wound healing and nude mouse tumor xenograft model assay. Further, the target of miR-221/222 was determined by luciferase reporter, western blot and gene rescue assay. The association of miR-221/222 with outcome was examined in fifty glioma patients.

Results: MiR-221/222 expression was significantly increased in high-grade gliomas compared with low-grade gliomas, and positively correlated with the degree of glioma infiltration. Over-expression of miR-221/222 increased cell invasion, whereas knockdown of miR-221/222 decreased cell invasion via modulating the levels of the target, TIMP3. Introduction of a TIMP3 cDNA lacking 3' UTR abrogated miR-221/222-induced cell invasion. In addition, knockdown of miR-221/222 increased TIMP3 expression and considerably inhibited tumor growth in a xenograft model. Finally, the increased level of miR-221/222 expression in high-grade gliomas confers poorer overall survival.
\end{abstract}

Conclusions: The present data indicate that miR-221 and miR-222 directly regulate cell invasion by targeting TIMP3 and act as prognostic factors for glioma patients.

Keywords: MiRNA, TIMP3, Glioblastoma, Cell invasion, Prognosis

\section{Background}

Glioblastoma multiforme (GBM) is the most frequent malignant primary brain tumor in adults. The hallmark of GBM is the widespread diffuse invasion of tumor cells into brain tissues. Owing to the aggressive infiltration into the surrounding normal brain, current therapies fail to control GBM $[1,2]$. With standard treatment, GBM patients have a median survival of 15.6 months, despite of surgery, radiation therapy and chemotherapy [3]. Thus, there is an urgent need to develop novel

\footnotetext{
* Correspondence: kang97061@yahoo.com

${ }^{\dagger}$ Equal contributors

${ }^{2}$ Department of Neurosurgery, Laboratory of Neuro-Oncology, Tianjin Neurological Institute, Tianjin Medical University General Hospital, Tianjin 300052, China

${ }^{3}$ Key Laboratory of Post-trauma Neuro-repair and Regeneration in Central Nervous System, Ministry of Education, Tianjin Key Laboratory of Injuries, Variations and Regeneration of Nervous System, Tianjin 300052, China Full list of author information is available at the end of the article
}

therapeutic approaches that focus on controlling the invasion of this malignancy.

MicroRNAs (miRNAs), a recently identified class of endogenous, small ( $\sim 22 \mathrm{nt})$, non-protein coding, singlestranded RNA molecules, negatively regulate proteincoding genes by base-pair matching with the 3' UTRs of mRNAs. MiRNAs are dysregulated in tumors and function as oncogenic miRNAs or as tumor suppressor miRNAs [4]. Growing evidence has indicated important roles for miRNAs in tumor invasion. MiR-21 is an important oncogenic miRNA that promotes cell invasion by regulating multiple genes, including PTEN, RECK and MARCKS in several kinds of cancers, such as glioma, ovarian epithelial carcinoma and prostate cancer [5-7]. In pancreatic cancer cells, miR-146a inhibits the invasive capacity with concomitant down-regulation of EGFR and the NF-kappaB regulatory kinase, interleukin 1 receptorassociated kinase 1 (IRAK-1) [8]. In addition, in glioma

\section{Biomed Central}

(c) 2012 Zhang et al.; licensee BioMed Central Ltd. This is an Open Access article distributed under the terms of the Creative Commons Attribution License (http://creativecommons.org/licenses/by/2.0), which permits unrestricted use, distribution, and reproduction in any medium, provided the original work is properly cited. 
cells, miR-7 binds to the EGFR 3'UTR and decreases cell invasiveness by suppressing translation of EGFR [9]. Over-expression of miR-146b inhibits glioma cell invasion by targeting matrix metalloproteinases (MMPs) [10]. However, there is little direct evidence to show the mechanism by which miR-221/222 controls glioma invasion.

In the current study, we demonstrate that high levels of miR-221/222 expression in gliomas confer highly aggressive invasion and poorer overall survival. Furthermore, knockdown of miR-221/222 decreased invasion capability, reduced tumor growth and up-regulated the expression of the target, TIMP3, whereas ectopic expression of miR-221/222 exhibited the opposite effects. These findings indicate that TIMP3 is a critical target of miR-221 and miR-222 and that these two miRNAs could be critical therapeutic targets and survival predictors in glioma.

\section{Materials and methods}

\section{Tissue samples and clinical data}

All patients underwent complete or partial surgical resection at Tianjin Huanhu Hospital and Beijing Tiantan Hospital. Fifty paraffin-embedded glioma specimens with clinical data were collected from January 2006 to June 2006, including 14 grade I-II tumors, 18 grade III tumors and 18 grade IV tumors. Twenty-two frozen glioma specimens, stored in liquid nitrogen, were collected from October 2009 to May 2010, including 9 grade I-II tumors, 6 grade III tumors and 7 grade IV tumors. Paraffin-embedded glioma specimens were used for MiRNA locked nucleic acid (LNA) in situ hybridization and immunohistochemistry, and frozen glioma specimen were used for Real-time PCR. This study was approved by the institutional review boards of the hospitals (the Ethics Committees of Tianjin Medical University, TMUhMEC 2009016) and written informed consent was obtained from all patients. Patients were followed by clinical and laboratory monitoring on a regular basis starting at definitive diagnosis. Disease-specific survival time was defined as the time from definitive diagnosis to disease-specific death.

\section{MR data acquisition}

Patients were scanned with a $3.0 \mathrm{~T}$ clinical MR imaging scanner (Magnetom Sonata; Siemens, Erlangen, Germany) equipped with the standard head coil according to a standardized hospital brain tumor imaging protocol. The scans used for this study included diffusion tensor imaging (DTI) and axial contrast-enhanced T1-weighted spin-echo scans. In low-grade gliomas, peritumoral edematous regions were selected by T2 and FLAIR. In every patient, we measure three times in peritumoral edematous different regions with the volume of ROI (5 pixels). DTI data were transferred to a workstation (Inspiron 8200; Dell, Round Rock, TX, USA) for analysis. Data processing was performed using DTIStudio (version 2.4; Johns Hopkins University, Baltimore, MD, USA).

\section{Cell culture and transfection}

Human U251 and LN229 glioblastoma cells, were obtained from the China Academia Sinica Cell Repository, Shanghai, China. The cells were maintained in Dulbecco's modified Eagle's medium (Gibco, Los Angeles, CA, USA) supplemented with $10 \%$ fetal bovine serum (Gibco), and were incubated at $37^{\circ} \mathrm{C}$ in a $5 \% \mathrm{CO}_{2}$ atmosphere. Cell transfection was performed using Lipofectamine 2000 (Invitrogen) according to the manufacturer's instructions.

\section{Plasmids and oligonucleotides}

2 '-OMe-oligonucleotides were chemically synthesized and purified by high-performance liquid chromatography (Gene Pharma, Shanghai, China). The sequences are: 2'-OMe -miR-221 (miR-221), 5'-AGCUACAUUGUCUGCUGGGU UUC-3'; 2'-OMe -miR-222 (miR-222), 5'-AGCUACAUCU GGCUACUGGGU-3'; 2'-OMe-As-miR-221 (As-miR-221), 5'-AGCUACAUUGUCUGCUGGGUUUC-3'; 2'-OMe-AsmiR-222 (As-miR-222), 5'-AGCUACAUCUGGCUACUGG GU-3'. Then As-miR-221 and/or As-miR-222 (200 pmol) were transfected using Lipofectamine 2000 (Invitrogen). Cells transfected with scrambled 2'-OMe oligonucleotides (scramble) were used as control. Wild-type TIMP3 lacking its 3' UTR in pCDNA3 was provided by Dr. J.Q. Cheng (Molecular Oncology, H. Lee Moffitt Cancer Center, Tampa, FL, USA).

\section{Real-time PCR}

MiRNA expression was analyzed using a Hairpin-itTM miRNA qPCR Quantitation Kit (GenePharma, Shanghai, China) and a DNA Engine Opticon 2 Two-color Realtime PCR Detection System (Bio-Rad, USA), according to the manufacturer's instructions. Cycle threshold (CT value) was acquired using the software provided by the manufacturer. U6 is used as the normalization gene in Real-time PCR. The real-time PCR data were analyzed using the ddCT method.

\section{Transwell assay}

Cells were detached and resuspended in serum-free medium. Cells $\left(1 \times 10^{5}\right.$ cells/well $)$ were then plated into Matrigel coated invasion chambers (Becton Dickinson) and allowed to invade for 24 hours. The remaining cells in the chambers were removed by cotton swabs and the invading cells on the lower surface of the chambers were fixed with $70 \%$ ethanol and then stained with hematoxylin. The number of invading cells was calculated by counting three different fields under a phasecontrast microscope. 


\section{Wound healing assay}

Cells were optimized to ensure a homogeneous and viable cell monolayer prior to wounding. Equal numbers of cells were seeded into six-well culture plates. According to the above methods, cells were transfected with As-miR-221/ 222 or miR-221/222. When the cell confluence reached about $90 \%$ at $48 \mathrm{~h}$ post-transfection, an artificial homogenous wound was created on the monolayer with a sterile plastic $100 \mu \mathrm{L}$ micropipette tip. After wounding, the debris was removed by washing the cells with serum-free medium. Migration of cells into the wound was observed after $36 \mathrm{~h}$. Cells that migrated into the wounded area or cells with extended protrusions from the border of the wound were photographed. A total of three areas were selected from each well at random, and three wells of each group were quantified in each experiment.

\section{Western blot}

Parental and transfected cells were washed with prechilled phosphate-buffered saline (PBS) three times. The cells were then solubilized in $1 \%$ Nonidet P-40 lysis buffer $(20 \mathrm{mM}$ Tris, $\mathrm{pH}$ 8.0, $137 \mathrm{mM} \mathrm{NaCl}, 1 \%$ Nonidet P-40, 10\% glycerol, $1 \mathrm{mM} \mathrm{CaCl} 2,1 \mathrm{mM} \mathrm{MgCl}_{2}, 1 \mathrm{mM}$ phenylmethylsulfonyl fluoride, $1 \mathrm{mM}$ sodium fluoride, $1 \mathrm{mM}$ sodium orthovanadate, and a protease inhibitor mixture). Total protein lysates were separated by SDSPAGE. The separated proteins were transferred to PVDF membranes. Blots were incubated with MMP2, MMP9 and TIMP3 (Santa Cruz) primary antibodies, followed by incubation with HRP-conjugated secondary antibody. The specific protein was detected using a super signal protein detection kit (Pierce). After washing with stripping buffer, PVDF membranes were reprobed with antibody against $\beta$-actin.

\section{ELISA assay}

Twenty-four hours post-transfection, cell culture media were collected and quantified for MMP2 and MMP9 levels using an MMP2 and MMP9-specific ELISA kit (R\&D Systems, Minneapolis, MN, USA), according to the manufacturer's protocol.

\section{MiRNA locked nucleic acid (LNA) in situ hybridization and immunohistochemistry}

Using an antisense locked nucleic acid (LNA/DNA) modified oligonucleotide probe, in situ hybridization was performed with fluorescence in situ hybridization kit (CYBRDI, China). MiR-221 and miR-222-LNA oligonucleotides with digoxigenin modification contained LNAs at five locations (underlined): 5' -GAAACCCAGCAGACAAT

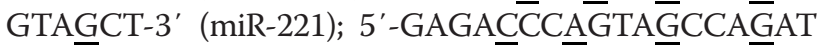
GTAGCT-3' (miR-222). LNA-miR-221 uses DIG modification and LNA-miR-222 uses BIO modification in 3' distal end. Sections were deparaffinized and deproteinated, and then prehybridized for $2 \mathrm{~h}$ in hybridization liquid in a humidified chamber ( $50 \%$ formamide, $5 \times$ SSC). The probes were added to the sections and incubated overnight at $40^{\circ} \mathrm{C}$ in a water bath. After washing 3 times, anti-digoxigeninrhodamine solution was added and incubated for $2 \mathrm{~h}$ at room temperature in the dark. Nuclei were counterstained with a DAPI karyotyping kit (Genmed, USA). After washing 3 times, sections were sealed and detected under a fluorescence microscope. In immunohistochemistry assay, sections were incubated with primary antibody (1:100 dilution) overnight at $4^{\circ} \mathrm{C}$, then incubated with a biotinylated secondary antibody (1:200 dilution) at room temperature for $1 \mathrm{~h}$, followed by incubation with $\mathrm{ABC}$-peroxidase reagent for an additional $1 \mathrm{~h}$. After washing with Tris-buffer, the sections were stained with DAB (30 mg 3,3 diaminobenzidine dissolved in $100 \mathrm{ml}$ Tris-buffer containing 0.03\% $\mathrm{H}_{2} \mathrm{O}_{2}$ ) for $5 \mathrm{~min}$, rinsed in water and counterstained with hematoxylin. The antibodies used in this study were to MMP2, MMP9 and TIMP3. The quantity and intensity scores were calculated such that a final score of $0-1$ indicated negative expression (-), 2-3 indicated weak expression $(+), 4-5$ indicated moderate expression $(++)$ and 6 indicated strong expression $(+++)$.

\section{Luciferase reporter assay}

The pGL3-WT-TIMP3-3'UTR-Luc reporter was created by the ligation of TIMP3 3' UTR PCR products into the XbaI site of the pGL3 control vector (Promega, USA). For the reporter assay, cells were cultured in 96-well plates and transfected with pGL3-TIMP3-3'UTR-Luc, and As-miR-221 and/or As-miR-221. Following $48 \mathrm{~h}$ incubation, luciferase activity was measured using a dualluciferase reporter system (Promega).

\section{Nude mouse tumor xenograft model and treatment}

U251 glioma cells were subcutaneously injected into 5week-old female nude mice (Cancer Institute of the Chinese Academy of Medical Science). When the tumor volume reached $50 \mathrm{~mm}^{3}$, the mice were randomly divided into four groups (eight mice per group). Each group was treated with $200 \mathrm{pmol}$ scramble oligo, AsmiR-221/222 in $10 \mu$ l Lipofectamine, miR-221/222 or PBS through local injection of the xenograft tumor at multiple sites (3-4 injected sites). The treatment was performed once every 3 days for 15 days. On day 28, tumors were harvested, fixed embedded in paraffin. The tumor volume was measured with a caliper twice a week, using the formula: volume $=$ length $\times$ width $^{2} / 2$.

\section{Statistical analysis}

Descriptive statistics, including mean and \pm SE, along with independent sample t-tests, and the Pearson correlation were used to determine significant differences. KaplanMeier analysis was employed to assess the survival rate of 
patients relative to expression levels of miR-221 and miR222. $\mathrm{P}<0.05$ was considered a significant difference.

\section{Results}

Correlation of miR-221/222 expression levels and glioma invasion

To evaluate tumor invasion in patients, we employed MR DTI, which is an MRI technique that can indirectly evaluate the integrity of the white matter by measuring water diffusion and its directionality. As shown in Figure $1 \mathrm{~A}$, the fractional anisotropy (FA) map and the threedimensional reconstruction of the fibers surrounding the glioma showed that GBM invaded and destroyed the corticospinal tract, while in grade II glioma the corticospinal tract was pushed around the tumor. For quantitative analysis of miR-221 and miR-222 in frozen tumor tissues of 22 patients, we performed real-time PCR. MiR-221 expression was significantly increased in high-grade gliomas compared with low-grade gliomas, and a similar trend for miR-222 was detected (Figure 1B). Recently, FA values from peritumoral edematous regions have been reported to be a quantitative index of brain invasion by glioma cells and to be negatively correlated with the degree of glioma infiltration [11]. The Pearson correlation showed that a significant negative correlation existed between FA values and miR-221 and miR-222 expression in these 22 gliomas $(\mathrm{R}=0.755, \mathrm{P}<0.005$ and $\mathrm{R}=0.612, \mathrm{P}<0.005$, respectively) (Figure $1 \mathrm{C}$ ). These findings suggest that miR-221/222 may have an important role in glioma invasion.

\section{Critical role of miR-221/222 in glioma cell invasion}

To further explore the role of miR-221/222 in cell invasion, we performed gain-of-function and loss-of-function analyses by over-expressing or suppressing miR-221/222 with As-miR-221/222 or miR-221/222, respectively.

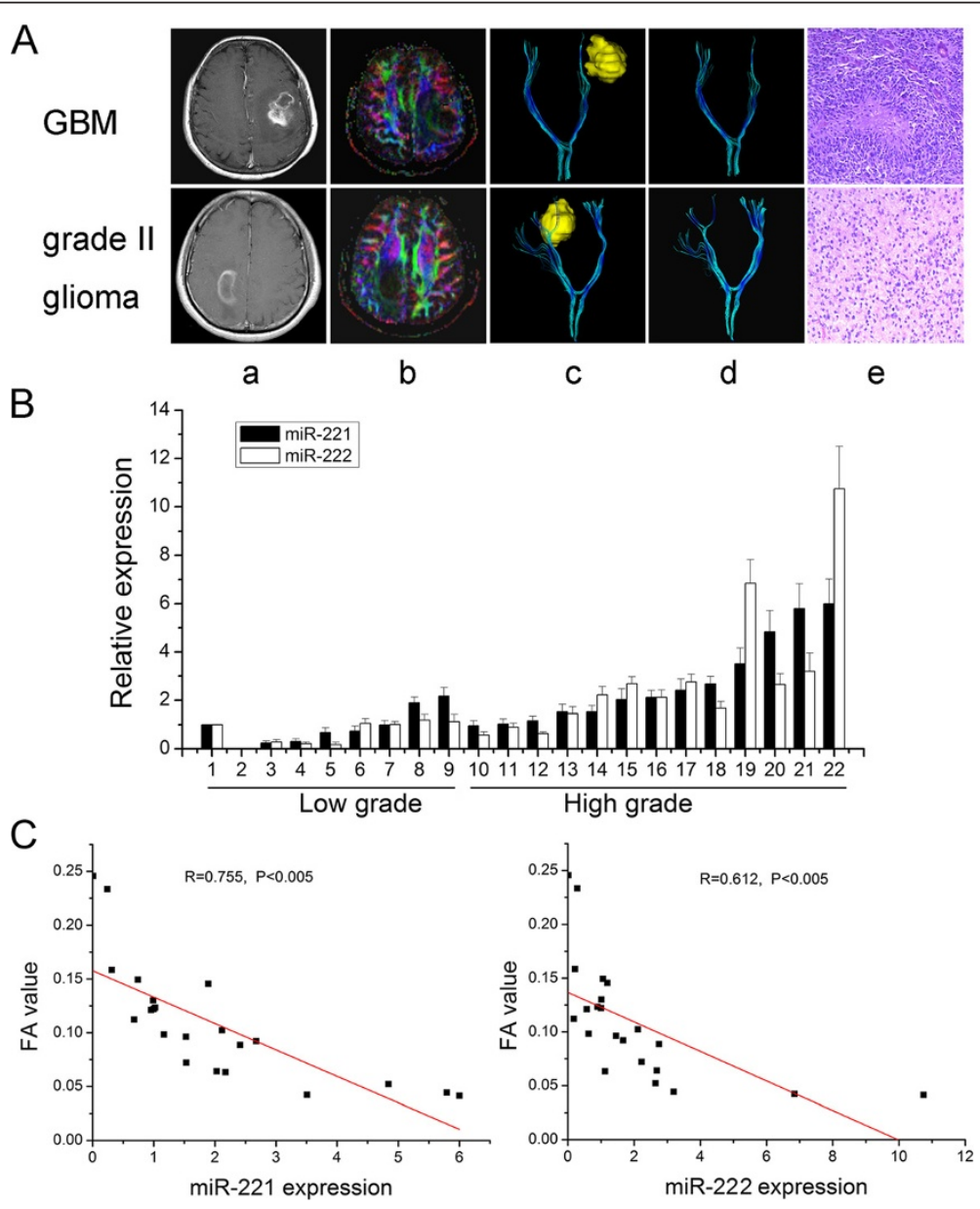

Figure 1 DTI and expression of miR-221 and miR-222 in gliomas. (A) DTI in glioma. a: contrast enhanced T1-weighted MRl; b: FA color map; $\mathrm{C}$ and d: three-dimensional reconstruction of the fibers surrounding the glioma (yellow); e: HE stain. (B) MiR-221 and miR-222 expression in 22 glioma tissues assayed by real-time PCR. (C) Correlation between FA values and miR-221 and miR-222 expression in these 22 gliomas by the Pearson correlation analysis. 
Interestingly, the transwell assay revealed that knockdown of miR-221/222 significantly decreased cell invasion potential compared with cells treated with scrambled oligonucleotide, whereas over-expression of
miR-221/222 increased cell invasion (Figure 2A). Consistent with the results of the transwell assay, in the wound healing assay, repression of miR-221/222 significantly inhibited cell migration, while over-expression of

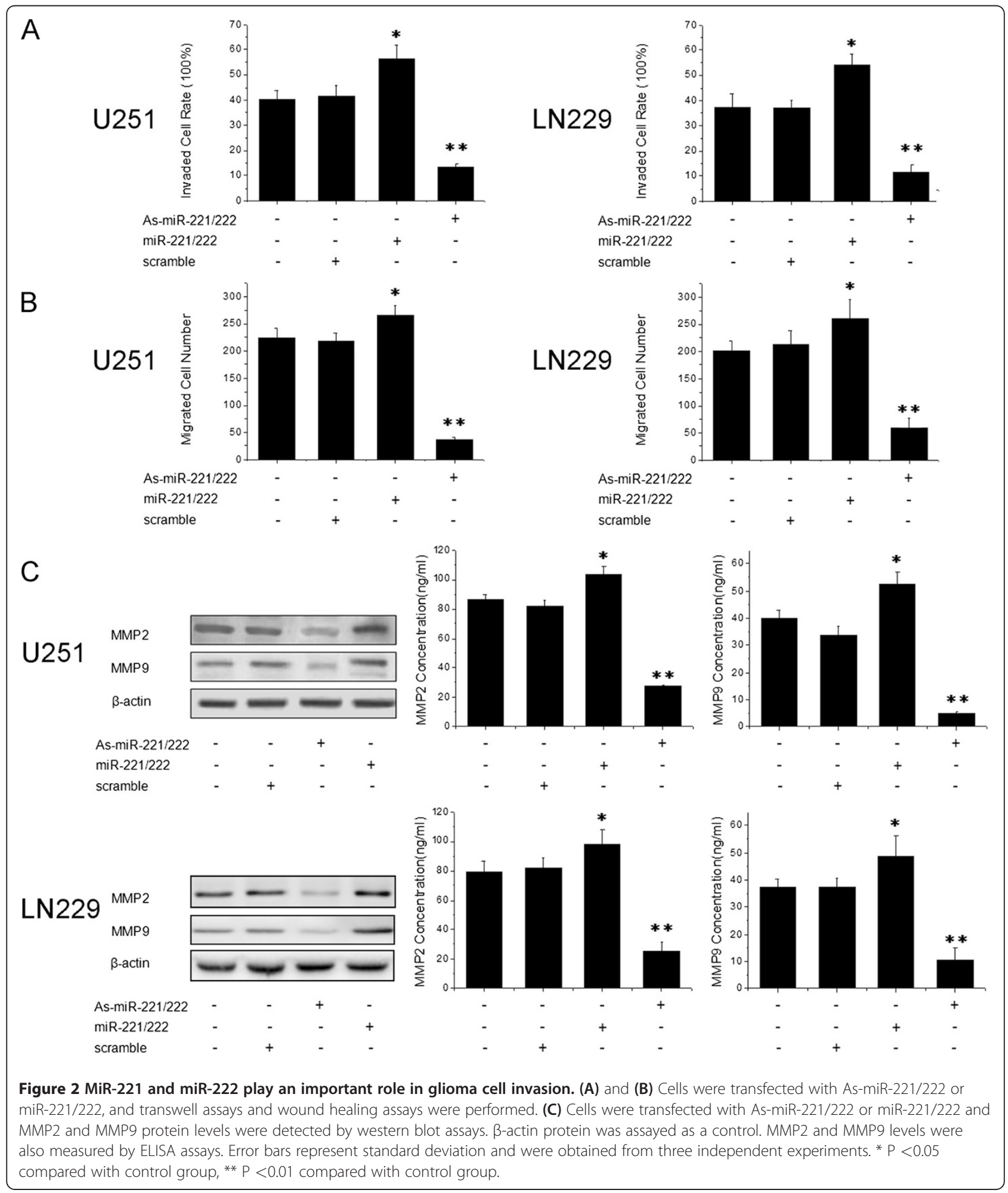


miR-221/222 increased migration in both U251 and LN229 cells (Figure 2B). To further explore the proteins relevant to invasion, the level of MMP2 and MMP9 were detected by Western blot and ELISA assays. The expression and secretion of MMP2 and MMP9 were significantly reduced in the As-miR-221/222 group but were up-regulated in the miR-221/222 group (Figure 2C). Therefore, miR-221 and miR-222 are required for glioma cell invasion.

\section{TIMP3 is a direct target of miR-221 and miR-222}

To determine the mechanism of action of miR-221 and miR-222 in glioma cell invasion, we performed a miRNA target search using TargetScan and found that the "seed sequence" of miR-221 and miR-222 matched the 3' UTR of the TIMP3 gene (Figure 3A), which has been evidenced in non small cell lung cancer cells.[12] To detect whether TIMP3 is indeed regulated by miR-221 and miR-222 in glioma cells, we knocked-down miR-221/222 and ectopically expressed miR-221/222 in U251 and LN229 cells. Western blot analysis showed that TIMP3 expression was up-regulated in cells with reduced levels of miR-221/222, whereas TIMP3 expression was down-regulated in cells over-expressing miR-221/222 (Figure 3B). In addition, we created pGL3-WT-TIMP3-3'UTR plasmids. Reporter assays revealed that a reduction of miR-221/222 triggered a marked increase of luciferase activity from pGL3-WT-
TIMP3-3'UTR (Figure 3C). These data indicate that miR221/222 can directly modulate TIMP3 expression by binding to the 3' UTR of TIMP3 in gliomas.

\section{Expression of TIMP3 overrides miR-221/222-induced invasion}

Having demonstrated TIMP3 to be a direct target of miR$221 / 222$ by our present and other previous studies [12], the importance of TIMP3 in miR-221/222-mediated cell invasion is still unclear. We next introduced TIMP3 lacking its 3' UTR into U251 and LN229 cells. Transwell and wound healing assays showed that cells transfected with TIMP3 had significantly reduced cell invasion and migration. However, expression of TIMP3 largely abrogated the effects of miR-221/222 on cell invasion (Figure 4A and B). Moreover, similar trends were observed in the expression of proteins relevant to invasion (TIMP3, MMP2 and MMP9) (Figure 4C). These results indicate that TIMP3 is a major target of miR-221 and miR-222 in regulating glioma cell invasion.

\section{As-miR-221/222 inhibits glioblastoma xenograft growth and induces TIMP3 up-regulation}

Because the levels of miR-221 and miR-222 are frequently elevated in glioblastoma and because they play an important role in cell survival, we further examined the effects of miR-

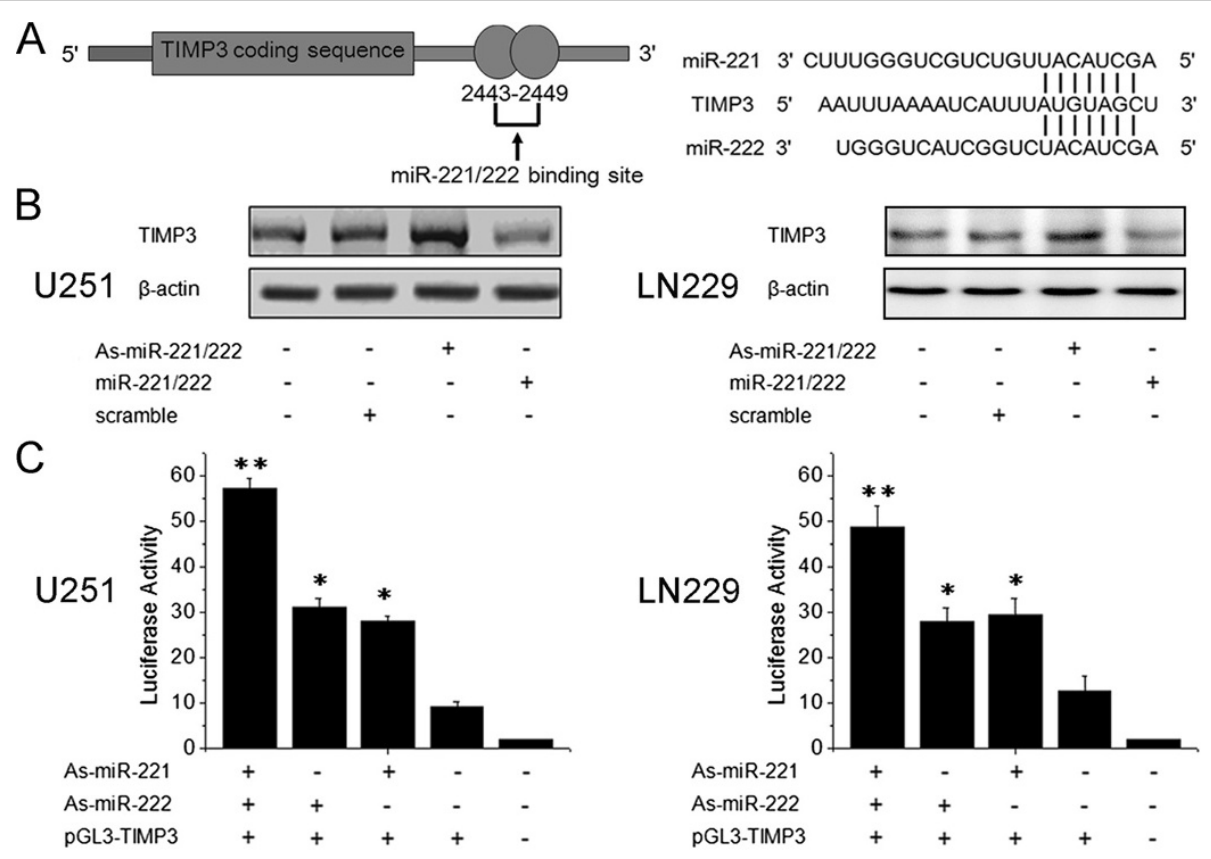

Figure 3 TIMP3 is a target for miR-221 and miR-222. (A) Schematic representation of the putative binding sites in the TIMP3 mRNA 3' UTR for miR-221 and miR-222 (the seed sequence (AGCUACAU) was identical, as shown). (B) Cells were transfected with As-miR-221/222 or miR-221/222 and TIMP3 protein levels were detected by western blot assays. $\beta$-actin protein was assayed as a control. (C) pGL3-TIMP3-3'UTR luciferase constructs were transfected into cells that were then treated with As-miR-221 and/or As-miR-222. Luciferase activity was determined $48 \mathrm{~h}$ after transfection. The ratio of normalized sensor to control luciferase activity is shown. Error bars represent standard deviation and were obtained from three independent experiments. ${ }^{*} P<0.05$ compared with control group, ${ }^{* *} P<0.01$ compared with control group. 


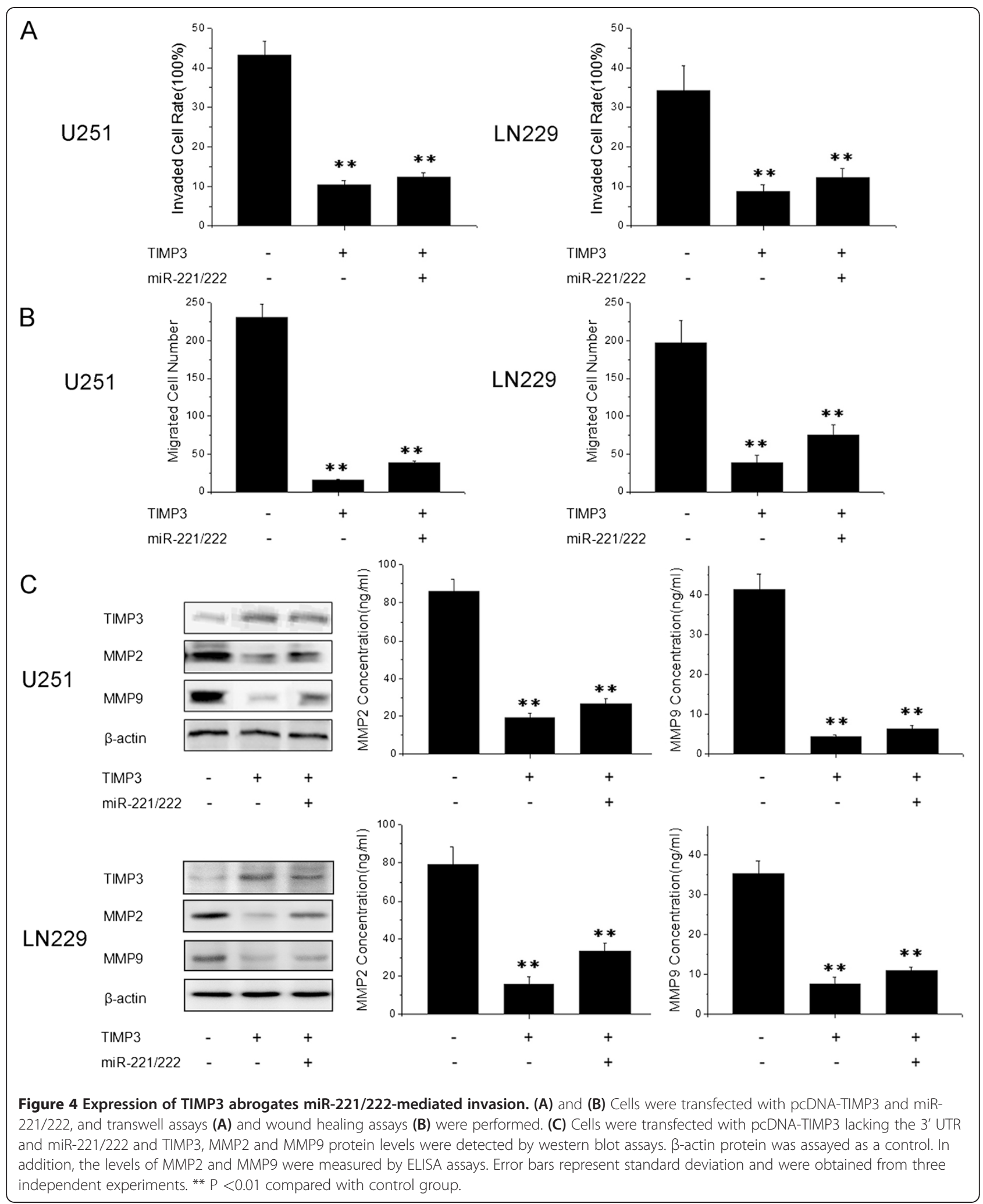

$221 / 222$ on tumor growth in a glioblastoma xenograft model. Reduction of miR-221/222 levels inhibited tumor growth in vivo, and over-expression of miR-221/222 slightly increased tumor growth (Figure 5A). Immunohistochemistry then revealed that TIMP3 levels were up-regulated in As-miR-221/222 treated tumors and down-regulated in 


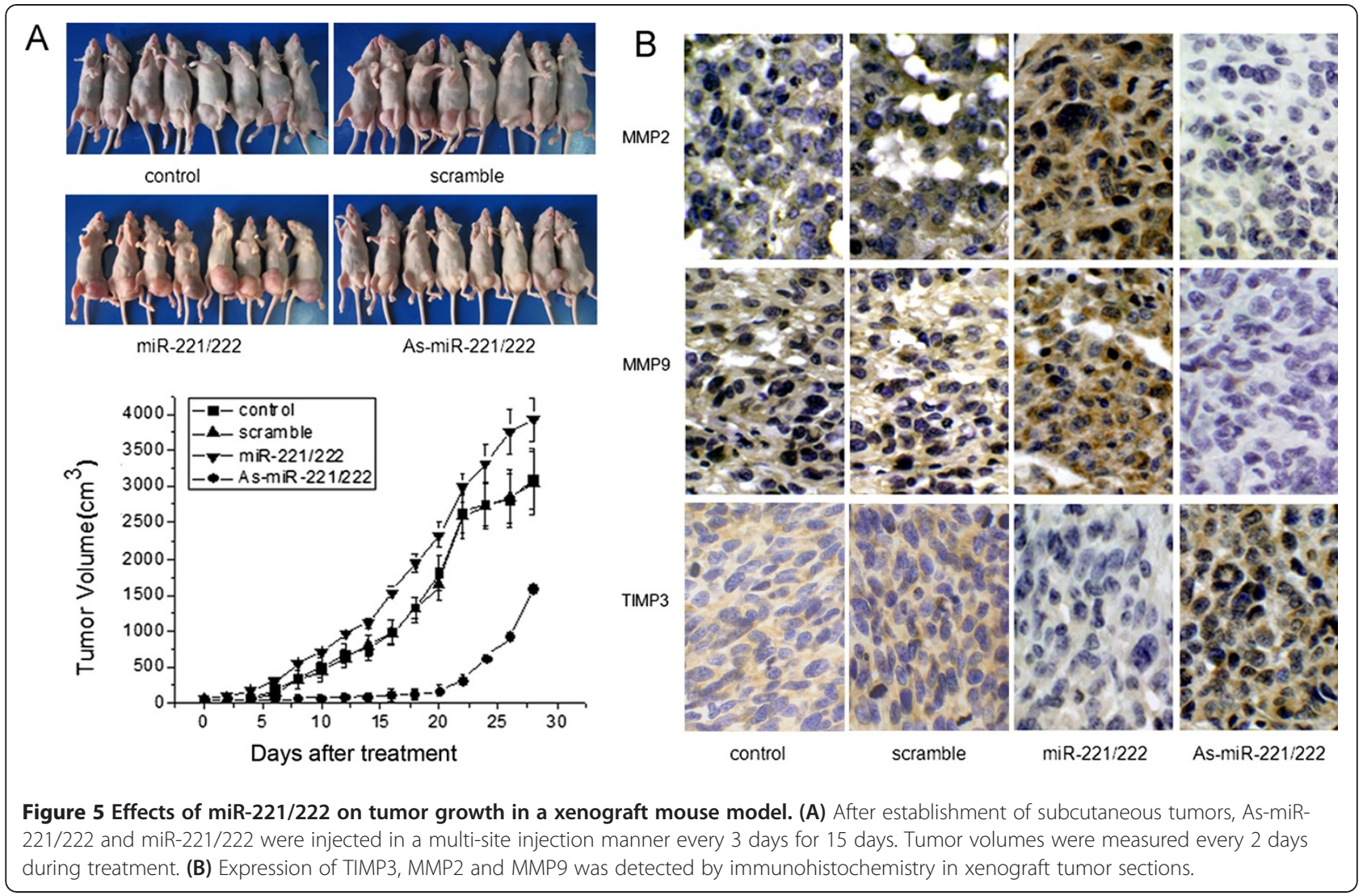

miR-221/222 treated tumors. In addition, levels of MMP-2 and MMP-9 expression in xenograft tumor sections confirmed the in vitro data (Figure 5B). Thus, As-miR-221/222 could be a therapeutic target for glioma intervention.

\section{Inverse correlation of expression of miR-221/222 and TIMP3 in glioma tissues}

Having demonstrated TIMP3 as a major target of miR$221 / 222$, we further investigated the correlation of between miR-221/222 and TIMP3 expression in gliomas. We examined 50 human paraffin-embedded glioma specimens with complete clinical data by miRNA-LNA in situ hybridization. Representative images of miR-221/222 are shown in Figure 6A. According to the expression profile of miR-221/222 and TIMP3, the tissue samples were categorized as low positive $(\leq 3)$ and high positive $(>3)$. There was an inverse relationship between miR221/222 and TIMP3 levels in glioma tissues.

Significant prognostic value of miR-221/222 in high-grade glioma

Our present data and previous studies have shown that miR-221/222 affects the behavior of glioma cells including invasion, proliferation, apoptosis and radioresistance, by regulating multiple targets, including TIMP3, p27, PUMA, and PTEN [13-15]. Thus, we reasoned that miR-221/222 should have a critical prognostic value for glioma patients (Figure 6B). We analyzed the overall survival of patients and found significant differences between the low positive group and the high positive group in high-grade gliomas. Kaplan-Meier survival curve analysis showed that a highly statistically significant correlation was observed between the overall survival and the expression levels of miR-221 $(\mathrm{P}=0.011)$ and miR-222 $(\mathrm{P}=0.020)$ in high-grade gliomas (Figure 6C). These data indicate that the miR-221/222 high positive cases have a markedly worse outcome.

\section{Discussion}

Glioma cell invasion along white matter tracts is believed to be one of the major reasons for the resistance to treatment. A quantitative analysis of glioma cell invasion would be of value for clinical applications and experimental studies. However, in clinical studies, measurements of tumor cell invasion remain controversial. DTI is a new MRI technique that can reveal abnormalities and destruction of white matter fibers and has been used to measure the degree of white matter damage in brain regions, thus providing the potential for quantitative analysis of tumor invasion [16]. Notably, we found that miR221 and miR-222 are associated with glioma cell invasion by integrating expression and DTI data. In high-grade glioma, DTI indicated a decrease and deflection of the 


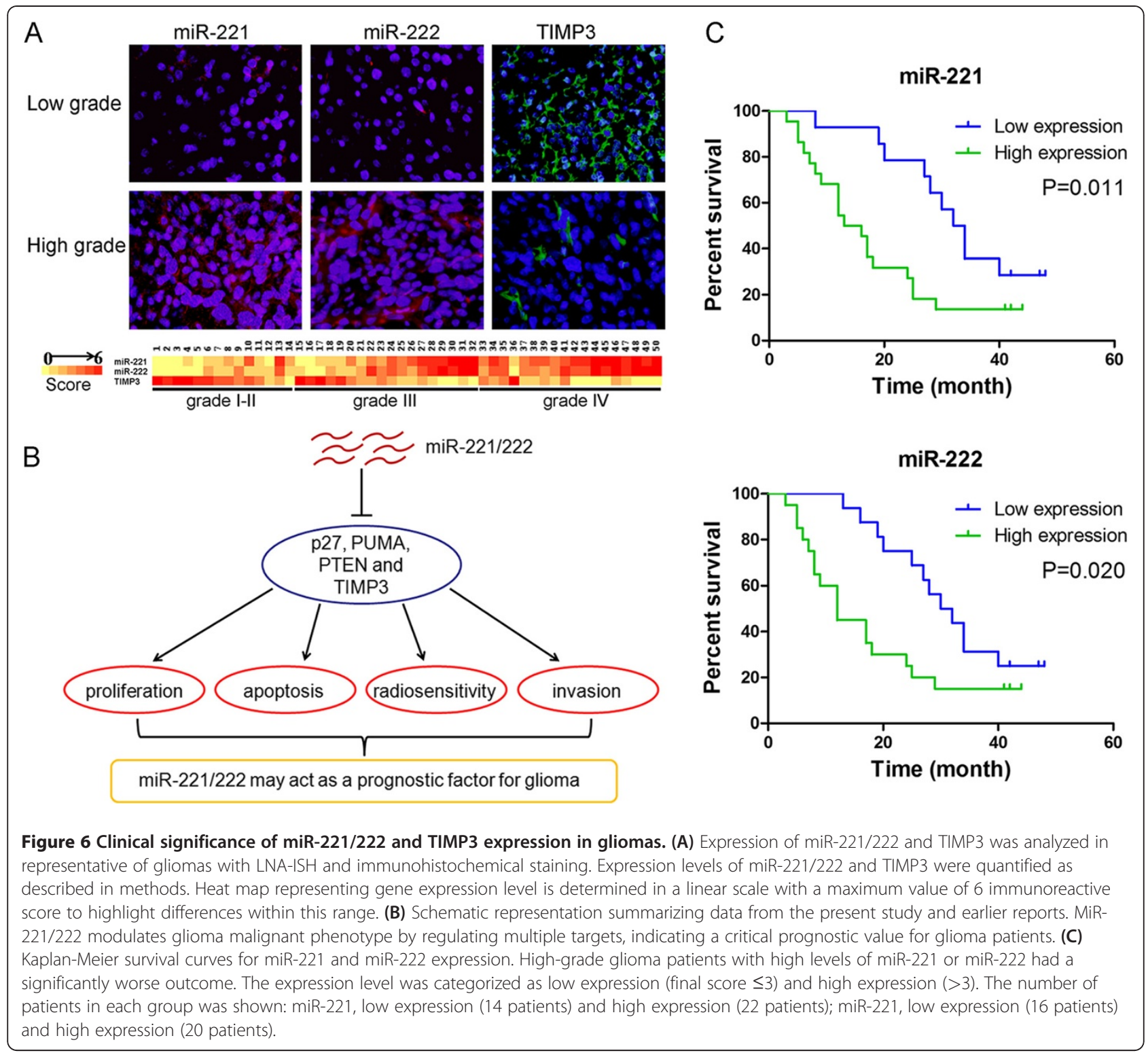

fibers surrounding the glioma that was associated with high levels of miR-221/222. To our knowledge, this is the first report combining analysis of a glioma invasion maker with DTI data.

MiR-221 and miR-222 share the same seed sequence, which are short, evolutionarily conserved regions through which miRNAs bind their target sites in mRNA 3' UTRs, indicating an important role in coordinated regulation and function. Several genes have been found to be common targets of these two miRNAs, such as p27, Bmf and PTEN $[13,17,18]$. Our recent data have shown that miR-221/222 inhibit cell apoptosis in human glioma cells by targeting the proapoptotic gene, PUMA. [14] Here, we demonstrated that miR-221/222 play an important role in the regulation of glioma invasion by directly targeting TIMP3, an inhibitor of MMPs. Among the MMPs, attention in human gliomas has focused on gelatinases (MMP2 and MMP9) [19]. A major mechanism for controlling the activity of MMPs in the pericellular space is mediated by the action of tissue inhibitors of metalloproteinases (TIMPs), which bind to active MMPs [20]. There are four metalloproteinase family members: TIMP1, TIMP2, TIMP3 and TIMP4. TIMP3 is unique within the TIMP family as it remains closely associated to the extracellular matrix after being secreted by the cell [21]. In our study, down-regulation of TIMP3 correlated with increasing malignancy in human gliomas. TIMP3 was also inversely correlated with miR-221/222 expression. Ectopic expression of TIMP3 inhibited U251 and LN229 glioma cell invasion through the inhibition of 
MMP2 and MMP9 expression and activity. Furthermore, expression of TIMP3 could largely override miR-221/ 222-mediated invasion. These results demonstrate that TIMP3 is a core target of miR-221/222 in glioma cell invasion.

The significance of miRNAs in prognostic determination has been shown in a variety of human cancers, such as lung cancer, pancreatic cancer, neuroblastomas and colon cancers [22-25]. However, few studies addressing the correlation between miRNA expression and glioma patient survival have been reported. Zhi et al. screened the expression profile of 200 miRNAs in a sample set consisting of 84 astrocytoma samples and 20 normal adjacent tissue samples and found that low level of miR-181b or miR-106a, or high level of miR-21, was significantly associated with poor patient survival [26]. Another study reported that the cumulative 5 year survival rate of glioma patients was $51.54 \%$ in the low miR-182expression group, whereas it was only $7.23 \%$ in the high miR-182-expression group. Moreover, multivariate Cox regression analysis indicated that miR-182 expression was an independent prognostic indicator for the survival of glioma patients [27]. In this study, our data suggest that a high miR-221/222 expression level is a valuable marker for pathological diagnosis and prognosis prediction in high-grade glioma; high miR-221/222 expression levels were significantly associated with poor survival in high-grade glioma patients as determined by Kaplan-Meier analysis. However, recent data showed that miR-221 and miR-222 were downregulated in GBM and neither prognostic nor predictive associations were found for miR221or miR-222 [28]. Thus, these controversial data needs to be further investigation.

\section{Conclusions}

In summary, our data demonstrate that miR-221/222 regulate glioma cell invasion by directly targeting TIMP3. We also provide direct evidence that high levels of miR221/222 expression are significantly associated with poorer overall survival. To conclude, our data suggest that miR$221 / 222$ could be intrinsic regulators of progression in glioma cells and could be used as potential targets and predictors of survival in this devastating disease.

\section{Competing interest}

The authors declare that they have no competing interests.

\begin{abstract}
Acknowledgments
This work is supported by the National Basic Research Project of China (2009CB918903 and 2010CB529406), National High Technology Research and Development Program 863 (2012AA02A508), the China National Natural Scientific Foundation (30901772), and the Natural Science Foundation of Tianjin Municipal Science and Technology Commission (10SYSYJC28800).
\end{abstract}

\section{Author details}

${ }^{1}$ Department of Radiation Oncology, Tianjin Huanhu hospital, Tianjin 300060, China. ${ }^{2}$ Department of Neurosurgery, Laboratory of Neuro-Oncology, Tianjin
Neurological Institute, Tianjin Medical University General Hospital, Tianjin 300052, China. ${ }^{3}$ Key Laboratory of Post-trauma Neuro-repair and Regeneration in Central Nervous System, Ministry of Education, Tianjin Key Laboratory of Injuries, Variations and Regeneration of Nervous System, Tianjin 300052, China. ${ }^{4}$ Department of Neurosurgery, The First Affiliated Hospital of Nanjing Medical University, Nanjing 210029, China. ${ }^{5}$ Department of Neurosurgery, Tiantan Hospital, Capital Medical University, Beijing 100050, China. ${ }^{6}$ Department of Radiology, Tianjin Huanhu Hospital, Tianjin 300060, China. ${ }^{7}$ Department of Neurosurgery, Tianjin Medical University General Hospital, Laboratory of Neuro-Oncology, Tianjin Neurological Institute, Tianjin 300052, China.

\section{Authors' contributions}

CZZ and JWH performed the experimental work. JXZ interpreted the data and helped to draft the manuscript. ZDS, YYW, LH and ML participated in the experiments. SZY, YPY, and JHW analyzed data. JT, PYP and CSK conceived of the study and participated in its design and coordination.

Received: 23 November 2011 Accepted: 8 June 2012

Published: 8 June 2012

\section{References}

1. Tate MC, Aghi MK: Biology of angiogenesis and invasion in glioma. Neurotherapeutics 2009, 6:447-457.

2. Chi A, Norden AD, Wen PY: Inhibition of angiogenesis and invasion in malignant gliomas. Expert Rev Anticancer Ther 2007, 7:1537-1560.

3. Stupp R, Hegi ME, Mason WP, van den Bent MJ, Taphoorn MJ, Janzer RC, Ludwin SK, Allgeier A, Fisher B, Belanger K, et al: Effects of radiotherapy with concomitant and adjuvant temozolomide versus radiotherapy alone on survival in glioblastoma in a randomised phase III study: 5-year analysis of the EORTC-NCIC trial. Lancet Oncol 2009, 10:459-466.

4. Calin GA, Croce CM: MicroRNA signatures in human cancers. Nat Rev Cancer 2006, 6:857-866.

5. Lou Y, Yang X, Wang F, Cui Z, Huang Y: MicroRNA-21 promotes the cell proliferation, invasion and migration abilities in ovarian epithelial carcinomas through inhibiting the expression of PTEN protein. Int J Mol Med 2010, 26:819-827.

6. Gabriely G, Wurdinger T, Kesari S, Esau CC, Burchard J, Linsley PS, Krichevsky AM: MicroRNA 21 promotes glioma invasion by targeting matrix metalloproteinase regulators. Mol Cell Biol 2008, 28:5369-5380.

7. Li T, Li D, Sha J, Sun P, Huang Y: MicroRNA-21 directly targets MARCKS and promotes apoptosis resistance and invasion in prostate cancer cells. Biochem Biophys Res Commun 2009, 383:280-285.

8. Li Y, Vandenboom TG 2nd, Wang Z, Kong D, Ali S, Philip PA, Sarkar FH: miR146a suppresses invasion of pancreatic cancer cells. Cancer Res 2010, 70:1486-1495

9. Kefas B, Godlewski J, Comeau L, Li Y, Abounader R, Hawkinson M, Lee J, Fine $H$, Chiocca EA, Lawler S, Purow B: microRNA-7 inhibits the epidermal growth factor receptor and the Akt pathway and is down-regulated in glioblastoma. Cancer Res 2008, 68:3566-3572.

10. Xia H, Qi Y, Ng SS, Chen X, Li D, Chen S, Ge R, Jiang S, Li G, Chen Y, et al: microRNA-146b inhibits glioma cell migration and invasion by targeting MMPs. Brain Res 2009, 1269:158-165.

11. Deng Z, Yan Y, Zhong D, Yang G, Tang W, Lu F, Xie B, Liu B: Quantitative analysis of glioma cell invasion by diffusion tensor imaging. J Clin Neurosci 2010, 17:1530-1536.

12. Garofalo M, Di Leva G, Romano G, Nuovo G, Suh SS, Ngankeu A, Taccioli C, Pichiorri $F$, Alder $H$, Secchiero $P$, et al: miR-221\&222 regulate TRAIL resistance and enhance tumorigenicity through PTEN and TIMP3 downregulation. Cancer Cell 2009, 16:498-509.

13. Zhang C, Kang C, You Y, Pu P, Yang W, Zhao P, Wang G, Zhang A, Jia Z, Han L, Jiang H: Co-suppression of miR-221/222 cluster suppresses human glioma cell growth by targeting p27kip1 in vitro and in vivo. Int J Oncol 2009, 34:1653-1660.

14. Zhang CZ, Zhang JX, Zhang AL, Shi ZD, Han L, Jia ZF, Yang WD, Wang GX, Jiang T, You YP, et al: MiR-221 and miR-222 target PUMA to induce cell survival in glioblastoma. Mol Cancer 2010, 9:229.

15. Zhang C, Kang C, Wang P, Cao Y, Lv Z, Yu S, Wang G, Zhang A, Jia Z, Han L, et al: MicroRNA-221 and -222 regulate radiation sensitivity by targeting the PTEN pathway. Int J Radiat Oncol Biol Phys 2011, 80:240-248. 
16. Cruz Junior LC, Sorensen AG: Diffusion tensor magnetic resonance imaging of brain tumors. Neurosurg Clin N Am 2005, 16:115-134.

17. Gramantieri L, Fornari F, Ferracin M, Veronese A, Sabbioni S, Calin GA, Grazi GL, Croce CM, Bolondi L, Negrini M: MicroRNA-221 targets Bmf in hepatocellular carcinoma and correlates with tumor multifocality. Clin Cancer Res 2009, 15:5073-5081.

18. Chun-Zhi Z, Lei H, An-Ling Z, Yan-Chao F, Xiao Y, Guang-Xiu W, Zhi-Fan J, Pei-Yu P, Qing-Yu Z, Chun-Sheng K: MicroRNA-221 and microRNA-222 regulate gastric carcinoma cell proliferation and radioresistance by targeting PTEN. BMC Cancer 2010, 10:367.

19. Levicar N, Nuttall RK, Lah TT: Proteases in brain tumour progression. Acto Neurochir (Wien) 2003, 145:825-838.

20. Jiang Y, Goldberg ID, Shi YE: Complex roles of tissue inhibitors of metalloproteinases in cancer. Oncogene 2002, 21:2245-2252.

21. Lamfers ML, Gianni D, Tung CH, Idema S, Schagen FH, Carette JE, Quax PH, Van Beusechem WW, Vandertop WP, Dirven CM, et al: Tissue inhibitor of metalloproteinase- 3 expression from an oncolytic adenovirus inhibits matrix metalloproteinase activity in vivo without affecting antitumor efficacy in malignant glioma. Cancer Res 2005, 65:9398-9405.

22. Yanaihara N, Caplen N, Bowman E, Seike M, Kumamoto K, Yi M, Stephens RM, Okamoto A, Yokota J, Tanaka T, et al: Unique microRNA molecular profiles in lung cancer diagnosis and prognosis. Cancer Cell 2006, 9:189-198.

23. Bloomston M, Frankel WL, Petrocca F, Volinia S, Alder H, Hagan JP, Liu CG, Bhatt D, Taccioli C, Croce CM: MicroRNA expression patterns to differentiate pancreatic adenocarcinoma from normal pancreas and chronic pancreatitis. JAMA 2007, 297:1901-1908.

24. Chen Y, Stallings RL: Differential patterns of microRNA expression in neuroblastoma are correlated with prognosis, differentiation, and apoptosis. Cancer Res 2007, 67:976-983.

25. Schetter AJ, Leung SY, Sohn JJ, Zanetti KA, Bowman ED, Yanaihara N, Yuen ST, Chan TL, Kwong DL, Au GK, et al: MicroRNA expression profiles associated with prognosis and therapeutic outcome in colon adenocarcinoma. JAMA 2008, 299:425-436.

26. Zhi F, Chen X, Wang S, Xia X, Shi Y, Guan W, Shao N, Qu H, Yang C, Zhang $Y$, et al: The use of hsa-miR-21, hsa-miR-181b and hsa-miR-106a as prognostic indicators of astrocytoma. Eur J Cancer 2010, 46:1640-1649.

27. Jiang L, Mao P, Song L, Wu J, Huang J, Lin C, Yuan J, Qu L, Cheng SY, Li J: miR-182 as a prognostic marker for glioma progression and patient survival. Am J Pathol 2010, 177:29-38.

28. Lakomy R, Sana J, Hankeova S, Fadrus P, Kren L, Lzicarova E, Svoboda M, Dolezelova H, Smrcka M, Vyzula R, et al: MiR-195, miR-196b, miR-181c, miR-21 expression levels and 0-6-methylguanine-DNA methyltransferase methylation status are associated with clinical outcome in glioblastoma patients. Cancer Sci 2011, 102:2186-2190.

doi:10.1186/1479-5876-10-119

Cite this article as: Zhang et al:: High level of miR-221/222 confers increased cell invasion and poor prognosis in glioma. Journal of Translational Medicine 2012 10:119.

\section{Submit your next manuscript to BioMed Central and take full advantage of:}

- Convenient online submission

- Thorough peer review

- No space constraints or color figure charges

- Immediate publication on acceptance

- Inclusion in PubMed, CAS, Scopus and Google Scholar

- Research which is freely available for redistribution 